\title{
Saul Fitelberg
}

Fitelberg ist ein Impresario jüdischer Herkunft aus Paris, der nach Pfeiffering kommt, um Leverkühn anzubieten, seinen Musikagenten zu sein. Der erste Teil des vorliegenden Kapitels konzentriert sich auf die Darstellung des jüdischen Impresarios Saul Fitelberg in Thomas Manns Doktor Faustus, indem für eine intersektionale Analyse dieser Figur plädiert und diese dementsprechend durchgeführt wird. Zugleich wird Saul Fitelberg mit der Mäzenin Frau von Tolna kontrastiert: Beide Figuren stehen für zwei Formen der Musikfinanzierung, nur die zweite, d. h. das Mäzenatentum, scheint sowohl im Roman als auch in Adornos Philosophie für die Neue Musik besser geeignet zu sein. Allerdings hat auch das Mäzenatentum seine Nachteile, da es beispielsweise nur eine scheinbare Autonomie ermöglicht. Über Formen der Musikfinanzierung und die Zukunft der Oper reflektiert auch Manzonis Doktor Faustus: Der Veroperung widmet sich der zweite Teil des vorliegenden Kapitels. Gegen eine exzessive Vermarktung des Musikwerkes scheint sich Manzonis Oper zu wehren, indem der Musikagent durch das Falsett als Lügner und durch den verstärkten monologischen Charakter seiner Rede vom Hauptgeschehen isoliert wird - und zugleich zu Reflexionen über die Bedingungen des Opernbetriebes anregt.

\subsection{Intersektionale Analyse der Figur}

Der „internationale[] Musik-Gewerbmann[] und Konzert-Unternehmer[]“ (DF: 575) Saul Fitelberg kommt im Jahr 1923 nach Pfeiffering. Ihm begegnet die 
Leser*innenschaft im 37. Kapitel: Das vorherige Kapitel schließt mit der Erwähnung von Schwerdtfegers und Leverkühns Aufenthalt im Schloss Tolna. ${ }^{1}$ Doktor Faustus als „Musiker-Roman“ (Ent: 25) übersieht kaum einen Aspekt des Lebens eines*r Komponist*in und geht folglich auch auf die Finanzierung und Verbreitung des künstlerischen Schaffens ein. Diesbezüglich finden sich sowohl in diesem Kapitel als auch in dem Kapitel über Frau von Tolna in prosopoietischer Form Auffassungen aus Adornos Musikphilosophie über die Finanzierung des Musikwerkes: Saul Fitelberg steht für die Welt der Impresari, Frau von Tolna für das Mäzenatentum. Beide verkörpern jeweils eine Seite der Welt außerhalb der „Einsamkeit“ (PhnM: 48) von Pfeiffering und des Kunstwerkes: Nicht zufällig unterstreicht Zeitblom, dass Frau von Tolna und Fitelberg zum „Kapitel ,Welt““

\footnotetext{
${ }^{1}$ Vgl. DF: 573 ff. Die Thomas Mann-Forschung hat sich lange Zeit bemüht, ein reales Vorbild für den jüdischen Impresario zu finden. Der Autor spricht in der Entstehung des „Doktor Faustus “ von einem ,ehemals in Paris tätigen Literatur- und Theateragenten S.C.“ (Ent: 156). Den vollständigen Nachnamen erwähnt seine Frau, die ihm auch die Person vorgeschlagen hatte, in ihren Memoiren: Collin. Collin steht ,freilich der Musik ferne“ (Ent: 156): Er dient Thomas Mann dazu, ,für den ,Weltmann“ ein Gesicht zu haben“ (ebd.). Laut Schmidt-Schütz wurden die Figur des jüdischen Impresarios und ihr Umfeld nicht nur vom Theateragenten inspiriert, sondern auch von den Memoiren Strawinskys, deren deutsche Ausgabe der Autor von Doktor Faustus konsultierte. Der in Russland geborene Komponist wird im Roman genauso wie Nietzsche und Schönberg nie explizit erwähnt. Dies spricht immerhin für seine Bedeutung, denn die nie genannten Personen spielen in Doktor Faustus eine wichtige Rolle, wie Thomas Mann am Beispiel von Nietzsche erläutert. Darüber hinaus widmet sich Adorno im zweiten Teil seiner Philosophie der Neuen Musik gerade Strawinsky (siehe PhnM: 127-200). Paris, wo Fitelberg lebt und arbeitet, war auch Strawinskys jahrelanger Lebens- und Arbeitsmittelpunkt. Darüber hinaus beschreibt der Impresario Premieren in der französischen Hauptstadt, die den Premieren Strawinskys ähnlich waren: ,[W]ährend des Abends [springt] alles von den Plätzen [...] und die Majorität brüllt: ,Insulte! Impudence! Bouffonnerie ignominieuse!', während sechs, sieben initiés, Erik Satie, einige Surrealisten, Virgil Thomson, aus den Logen rufen:,Quelle précision! Quel esprit! C'est divin! C'est suprême! Bravo! Bravo!““ (DF: 582). Die Fitelberg-Episode hat weiters dem Autor zufolge ,etwas von der munteren Zweideutigkeit und Theaterwirksamkeit einer Riccaut de la Marlinière-Szene" (Ent: 156): Harry L. Stout unterstreicht in seinem Aufsatz die Ähnlichkeiten zwischen Lessings Darstellung von de la Marlinière in Minna von Barnhelm und der Thomas Manns vom exzentrischen Impresario. Siehe Mann, Katia: Meine ungeschriebenen Memoiren. Hrsg. v. Elisabeth Plessen und Michael Mann. Frankfurt am Main: Fischer 1976, S. 83; Schmidt-Schütz: Doktor Faustus zwischen Tradition und Moderne, S. 151 u. 156-159; Strawinsky, Igor: Erinnerungen. Übers. v. Richard Tüngel. Zürich/Berlin: Atlantis 1937 [Paris 1935]; Stout, Harry L.: Lessing's Riccaut and Thomas Mann's Fitelberg. In: The German Quarterly 36 (1963) H. 1, S. 24-30; Ent: 29.
} 
(DF: 575) seiner Biographie gehören. ${ }^{2}$ Die Art und Weise, wie Fitelberg und Frau von Tolna Leverkühn jeweils anbieten, sich um die Verbreitung und Finanzierung seines Werkes zu kümmern, ist jedoch unterschiedlich. Unterschiedlich sind auch die Bedingungen des jeweiligen Unterstützungsverhältnisses, denn Fitelberg fordert z. B. die körperliche Präsenz Leverkühns bei den Aufführungen seiner Werke, während das Mäzenatentum à la Frau von Tolna zur Einsamkeit zwingt und folglich für die Popularität des*r Komponist*in ein Hindernis darstellt. Mit diesen zwei Formen der Musikfinanzierung - die Darstellung von Fitelberg und Frau von Tolna regt zu ihrer Reflexion an - beschäftigt sich das vorliegende Kapitel: Das intermediale telling von Doktor Faustus beschränkt sich nicht nur auf die Beschreibung von Musikstücken und Musikerfiguren, sondern schließt auch die finanziellen Bedingungen der Musikproduktion und seiner Akteur*innen ein. ${ }^{3}$

\subsubsection{Der jüdische Impresario}

Saul Fitelberg wird im Roman zugleich als Jude und als Impresario dargestellt. Leverkühn und Zeitblom gegenüber stellt er sich mit folgenden Worten vor: ,[I]ch bin Jude, müssen Sie wissen: Fitelberg, das ist ein ausgesprochen mieser, polnisch-deutsch-jüdischer Name“ (DF: 580), sagt er und ergänzt eine Seite danach: ,[D]enn wie Sie mich da sehen, bin ich Impresario, bin es von Geblüt, bin es notwendiger Weise“ (DF: 581). Bereits diese Zitate eröffnen die Möglichkeit einer narratologisch-intersektionalen Analyse dieser Figur, da in der Darstellung die beiden Identitätskategorien der Ethnizität und der Klasse bzw. des beruflichen Status kaum voneinander zu trennen sind: In der Narration werden ,soziokulturelle[] Differenzen“4 konstruiert, Interdependenzen von Identitätskategorien werden erzählt und der literarische Text als „Laboratorium der Gesellschaft“5

\footnotetext{
${ }^{2} \mathrm{Zu}$ Frau von Tolna siehe DF: 567: „,[D]a es sich um eine Frau von Welt handelte, welche dem Einsiedler von Pfeiffering auch wirklich die Welt repräsentierte“.

${ }^{3}$ Siehe Lubkoll: Musik in Literatur: Telling.

${ }^{4}$ Nünning, Vera u. Ansgar Nünning: ,Gender'-orientierte Erzähltextanalyse als Modell für die Schnittstelle von Narratologie und intersektioneller Forschung? Wissenschaftsgeschichtliche Entwicklung, Schlüsselkonzepte und Anwendungsperspektiven. In: Klein, Christian u. Falko Schnicke (Hrsg.): Intersektionalität und Narratologie. Methoden Konzepte - Analysen. Trier: WVT 2014, S. 33-60, hier: S. 46.

${ }^{5}$ Blome, Eva: Erzählte Interdependenzen. Überlegungen zu einer kulturwissenschaftlichen Intersektionalitätsforschung. In: Pohl, Peter C. u. Hania Siebenpfeiffer (Hrsg.): Diversity Trouble. Vielfalt - Gender - Gegenwartskultur. Berlin: Kadmos 2016, S. 45-67, hier: S. 60 .
} 
eröffnet „ein[en] Raum der Reflexion“6 über die damalige politische und kulturelle Situation sowie die damaligen sozialen Kategorien im europäischen Raum, wie es im Folgenden aufgezeigt wird.

Fitelberg hat an alles gedacht, um auf Leverkühn den besten Eindruck zu machen: $\mathrm{Zu}$ Leverkühns Wohnort fährt er in einem Auto „samt Chauffeur" (DF: 576), gibt Frau Schweigestill eine „Besuchskarte“ (DF: 576) und erscheint „sommerlich elegant“ (DF: 578) gekleidet, ,in einen auf Taille gearbeiteten, bläulich gestreiften Flanellanzug, zu dem er Schuhe aus Leinen und gelbem Leder trug“" (DF: 578). Zeitblom beschreibt ihn als

ein[en] wohl vierzigjährige[n] fette[n] Mann, nicht bauchig, aber fett und weich von Gliedern, mit weißen, gepolsterten Händen, glattrasiert, vollgesichtig, mit Doppelkinn, stark gezeichneten, bogenförmigen Brauen und lustigen Mandelaugen voll mittelmeerischen Schmelzes hinter der Hornbrille. (ebd.)

Die Erzählinstanz präsentiert ihn dem obigen Zitat entsprechend als gepflegten, vielleicht wohlhabenden Geschäftsmann und die antisemitischen Stereotypen sind in diesem Zusammenhang nur schwer zu leugnen. ${ }^{7}$ Mehr noch, Zeitblom und Frau Schweigestill bezeichnen Fitelberg in kurzer Zeit als „Dummkopf“ (DF: 579) und „,spinnerten Uhu“ “ (DF: 576): Der Erzähler lässt hier Wertungen in die Narration einfließen, die zweifellos nicht darauf abzielen, Sympathie für Fitelberg zu hegen. Aufgrund der gleichzeitigen Darstellung von Fitelberg als Impresario und als Jude bleibt unklar, welche der beiden Kategorien Zeitblom zu diesen Wertungen führt. Intersektional betrachtet scheint eine Trennung auch nicht sinnvoll, denn es sind eben die „Überkreuzungen, Überlappungen bzw. Interdependenzen von Identitätskategorien“ ${ }^{8}$ die auch hier zu einer gewissen Unsichtbarkeit von sozialen Ungleichheiten und Diskriminierungsprozessen beitragen. ${ }^{9}$ Bevor Fitelberg die Möglichkeit hat, sich selbst zu präsentieren, werden zwei Aspekte durch die Erzählinstanz angedeutet: Es handelt sich bei ihm um keinen Künstler, sondern um eine Figur, die mit Geld arbeitet und wahrscheinlich jüdischer Herkunft

\footnotetext{
${ }^{6} \mathrm{Ebd}$.

${ }^{7}$ Zum möglichen Antisemitismus Zeitbloms vgl. 7.1.2.

${ }^{8}$ Blome: Erzählte Interdependenzen, S. 46, Herv. i. O.

${ }^{9}$ Siehe ebd. 49; Crenshaw, Kimberle: Demarginalizing the Intersection of Race and Sex: A Black Feminist Critique of Antidiscrimination Doctrine, Feminist Theory, and Antiracist Politics. In: University of Chicago Legal Forum (1989), S. 139-167; zum Begriff ,soziale Ungleichheit' siehe Schnicke, Falko: Terminologie, Erkenntnisinteresse, Methode und Kategorien - Grundfragen intersektionaler Forschung. In: Klein u. Schnicke (Hrsg.): Intersektionalität und Narratologie, S. 1-32, hier: S. 4 Fußnote 10.
} 
ist. Zeitblom und Frau Schweigestill sehen in Fitelberg eine potentielle Gefahr für ihr alltägliches Leben und ihre moralischen Sitten: Frau Schweigestill schließt die Tochter in ihrem Zimmer ein, Zeitblom ist froh, ,zu Adrians Bedeckung zur Stelle zu sein“ (DF: 577). ${ }^{10}$

Die Fitelberg-Episode ist quasi ein Monolog, denn - so Stout - „[d]uring the ensuing conversation the visitor does most or all of the talking, much of which is about himself", ${ }^{11}$ was die Nähe zum möglichen dramatischen Hypotext (Lessing) noch weiter betonen könnte.

Wodurch zeichnet sich ein Impresario bzw. eine Impresaria aus ${ }^{12}$ Jutta Toelle listet unter den Eigenschaften einer Musikagentin bzw. eines Musikagenten „Risikobereitschaft, Überredungskünste, hohes Selbstvertrauen“13 auf. Eine schnelle Lektüre dieser Episode reicht, um dem Impresario aus Paris diese Eigenschaften

\footnotetext{
${ }^{10}$ Thomas Mann schreibt in der Entstehung, ,daß die Gefahr einer antisemitischen Mißdeutung meiner jüdischen Riccaut-Figur, bei aller sympathischen Drolerie [...], nicht ganz von der Hand zu weisen ist" (Ent: 156). Diese Episode hat zusammen mit der Schilderung des Dr. Chaim Breisacher in Doktor Faustus und u. a. der Novelle Wälsungenblut $\mathrm{zu}$ einer heftigen Kontroverse über den möglicherweise versteckten Antisemitismus des Autors geführt. Die Polemik begann kurz nach der Veröffentlichung von Doktor Faustus und dem Ende des Zweiten Weltkriegs. Siehe Manns Eintrag am 31. Dezember 1948 in den Tagebüchern: „Der Angriff auf mich wegen Antisemitismus im Faustus u. anderwärts nicht in ,Commentary", sondern in einem Washingtoner ,Congressional Weekly“. Dumm und harmlos" (TB 3: S. 348). Todd Kontje glaubt beispielsweise, Manns Ambivalenz gegenüber den Juden spiegle seine „ambivalence towards himself“ wider: Als Kind einer nicht rein deutschen Familie und Mensch unklarer sexueller Orientierung sind für ihn die Juden ,people with whom he clandestinely identifies“. Kontje, Todd: Thomas Mann's Wälsungenblut: The Married Artist and the „Jewish Question“. In: PMLA 123 (2008) H. 1, S. 109-124, hier: S. 120. Diese Polemik lässt eine gewisse biographical fallacy nicht übersehen.

${ }^{11}$ Stout: Lessing's Riccaut, S. 24. Siehe auch Fußnote 1.

${ }^{12}$ Rosselli erläutert, Impresari seien selten weiblichen Geschlechts gewesen, jedoch ist in dem Grove-Eintrag nicht zu lesen, dass es keinerlei Beweise für die Existenz weiblicher Musikagentinnen gibt. Daher werden auch diesbezüglich genderneutrale Formulierungen präferiert; die Pluralform des Wortes hat im heutigen Sprachgebrauch des Italienischen jedoch nur Männer im Blick. An dieser Stelle sei allerdings erwähnt, dass die vorliegende Arbeit keineswegs den Zweck einer musikhistorischen Rekonstruierung unter gender-Aspekten des Impresario/Impresaria-Berufs verfolgt. Siehe Rosselli, John: Impresario (Ger. Schauspieldirektor). In: Grove Music Online. Zuerst veröffentlicht 01.12.1992, online veröffentlicht 2002.<https://doi.org/10.1093/gmo/9781561592630.article.O007223 >(letzter Zugriff: 21.08.2020); Toelle: Oper als Geschäft; Rosselli, John: The Opera Industry in Italy from Cimarosa to Verdi: The Role of the Impresario. Cambridge: Cambridge University Press 1984.

${ }^{13}$ Toelle, Jutta: Oper als Geschäft. Impresari an italienischen Opernhäusern 1860-1900. Kassel: Bärenreiter 2007, S. 21.
} 
zuordnen zu können. Seine Risikobereitschaft äußert sich z. B. dadurch, dass er sein Geld riskieren würde, um die Verbreitung von Leverkühns Musik zu unterstützen, obwohl diese nicht besonders zugänglich ist. In der Vergangenheit trug Fitelberg bereits das finanzielle Risiko eines ,exklusive[n] Boulevard-Theater[s]“ (DF: 580) namens „Théâtre des fourberies gracieuses“ (ebd.). Das Projekt war gescheitert, denn - wie er im Roman erklärt - ,[m]it James Joyce, Picasso, Ezra Pound und der Duchesse de Clermont-Tonnère als Publikum allein kommt man nicht aus" (ebd.). Toelle berichtet zudem davon, dass Musikagent*innen Theaterkonten zu ihren Gunsten verfälschten. ${ }^{14}$

Was Fitelbergs Überredungskünste angeht, so sind diese schwer zu leugnen: Er ist sowohl ,,a name dropper“ ${ }^{15}$ wie die Erzählung von dem Boulevard-Theater deutlich macht als auch ,a skilful flattere[r]“. ${ }^{16}$ Es könnte wohl sein, dass er mit dem Auto und elegant gekleidet kommt, weil er den Eindruck verstärken will, dank seiner Arbeit reich geworden zu sein. Sein Monolog, in dem er sich als Selfmademan vorstellt, zeugt von seinem großen Selbstvertrauen. ${ }^{17}$ Geboren ist er in „Ljublin mitten in Polen, von wirklich ganz kleinen jüdischen Eltern“ (DF: 530), lebt aber seit zwanzig Jahren in Paris und hat auch ,an der Sorbonne philosophische Vorlesungen gehört“ (DF: 580). Schnell lernte er, sich ,eine Smoking-Schleife zu binden“ (DF: 581) und „unter der crème de la crème zu bewegen" (DF: 581). Dass er aus keiner wohlhabenden Familie stammt, entspricht dem Lebenslauf verschiedener Impresari. ${ }^{18}$ Sein Selbstvertrauen geht aus den bisher erwähnten Passagen des Romans deutlich hervor.

Nun lohnt es sich zu untersuchen, welchen Stellenwert Impresari im musikalischen Bereich hatten. Die berühmte Satire auf die italienische Oper von

\footnotetext{
${ }^{14}$ Vgl. ebd., S. 13.

${ }^{15}$ Stout: Lessing's Riccaut, S. 25.

${ }^{16}$ Ebd., S. 26.

${ }^{17}$ Marquardt ist der Auffassung, Fitelberg sei ein assimilierter Ostjude. Vgl. Marquardt, Franka: Der Manager als Sündenbock. Zur Funktion des jüdischen Impresario Saul Fitelberg in Thomas Manns „Doktor Faustus“. In: Zeitschrift für Germanistik 14 (2004) H. 3, S. 564-580, hier: S. 573.
}

${ }^{18}$ Siehe Toelle: Oper als Geschäft, S. 21. 
Benedetto Marcello stellt sie als Lügner und Scharlatanen vor. ${ }^{19}$ Im Kapitel „Agl'Impresari“20 liest man: ${ }^{21}$

Pagherà i Viaggi l'Impresario alle Virtuose forastiere, perché vengano sicuramente, promettendogli buon Alloggio vicino al Teatro, Cibarie, Biancaria, etc. e le alloggerà poi in qualche picciola cucinetta (pur che sia vicina al Teatro) ripiena però di tutte le suddette cose [...].

Der*die Musikagent*in hält also seine Versprechen nicht ein: Lügen sind Bestandteil seiner bzw. ihrer Marketingstrategie. Tatsächlich etablierte sich diese Vorstellung so sehr, dass oft die Impresari als Sündenböcke für „die Misere der Opernindustrie“"22 fungierten. Ende des 19. Jahrhunderts, als die Welt der italienischen Oper in eine Krise geraten war, hielt man sie für die „Ausbeuter der Kunst und der Künstler" 23 par excellence. In der Tat starben sie aber nicht selten bankrott, weil sie sich so sehr für Musik einsetzten, dass sie Geldaspekte nicht ausreichend berücksichtigten. ${ }^{24}$

In Doktor Faustus passiert nichts anderes: „Im Verlauf seines buchstäblich pausenlosen ,Geplappers ' wird aus dem Teufel mit Zaubermantel ein veritabler Sündenbock“" ${ }^{25}$ erklärt Marquardt. Im ersten Teil des Gesprächs stellt sich Fitelberg vor und versucht, Leverkühn davon zu überzeugen, ihn als Musikagenten zu engagieren. ${ }^{26}$ Sein Angebot erweckt jedoch kein Interesse bei dem Komponisten.

\footnotetext{
${ }^{19}$ Benedetto Marcello (1686 Venedig - 1739 Brescia) war Komponist, Dichter und Theoretiker: „1720 gab Marcello anonym die beißende Satire Il teatro alla moda in den Druck, ein Angriff gegen die Unsitten an den Opernhäusern [...]“. Bizzarini, Marco: Art. Marcello, Benedetto. In: MGG Online. Zuerst veröffentlicht 2004, online veröffentlicht 2016. < https://www.mgg-online.com/mgg/stable/51086 > (letzter Zugriff: 21.08.2020).

${ }^{20}$ Marcello, Benedetto: Il teatro alla moda. Rom: Castelvecchi 1993 [Venedig 1720], S. 66. ${ }^{21}$ Ebd., S. 69, Herv. i. O. Die Übersetzung lautet: „Auswärtigen Sängerinnen zahle der Intendant die Anreise, damit diese auch wirklich kommen. Er verspreche ihnen eine gute Unterkunft unweit des Theaters, diverse Leckereien, Wäsche etc. und quartiere sie dann (auf jeden Fall in Theaternähe) in einer winzigen Besenkammer ein, die allerdings mit o. g. Aufmerksamkeiten vollgestopft ist“. Marcello, Benedetto: Das neumodische Theater. Übers. u. hrsg. v. Sabine Radermacher. Heidelberg: mkverlag 2001. Die Übersetzung ist leider nicht sehr präzise (siehe z. B. die Verwechslung zwischen Intendanten und Impresario und die Übersetzung von „cucinetta“, also einer Art winzige Einzimmerwohnung, als „Besenkammer“).

${ }^{22}$ Toelle: Oper als Geschäft, S. 64.

${ }^{23}$ Ebd.

${ }^{24}$ Siehe ebd.

${ }^{25}$ Marquardt: Der Manager als Sündenbock, S. 580.

${ }^{26}$ Vgl. DF: 576-585.
} 
Vor allem ist Leverkühn nicht bereit, die Bedingungen einer solchen Zusammenarbeit, auf die im Folgenden noch näher eingegangen wird, zu akzeptieren: Zwar kann sich Fitelberg damit abfinden, dass Leverkühn seine Kompositionen nicht dirigieren und am Klavier begleiten möchte, die conditio sine qua non besteht aber darin, dass er bei den Aufführungen seiner Werke anwesend ist. ${ }^{27}$ „Dies allerdings ist Bedingung“ (DF: 584), sagt Fitelberg: ,particulièrement à Paris“ (ebd.) sei Leverkühns ,persönliches Erscheinen [...] unerläßlich“ (ebd.). Im Laufe seines Monologs wird sich Fitelberg aufgrund der zurückhaltenden Reaktionen des Komponisten seines Scheiterns bewusst.

Statt sich gleich zu verabschieden, erlaubt er sich eine kleine „Rache“. Zuerst kritisiert er das Verhalten Leverkühns, der nichts von anderen Künstler*innen wissen und an den Aufführungen seiner Werke nicht teilnehmen will, indem er es als „personalistischen Einsamkeitshochmut“ (DF: 587) bezeichnet. Dann überlegt er laut, ob das Scheitern seines Besuchs überhaupt eine Enttäuschung für ihn sei. ${ }^{28}$ Danach reflektiert er darüber hinaus noch über den ,deutschen Charakter“ (DF: 590) und die Vorteile einer Mediation durch die Juden. Fitelberg hat als Ostjude Mut gezeigt, weil er - darauf weist Marquardt hin - an einem Schabbat, im Krisenjahr 1923, in Bayern aufgetaucht ist. ${ }^{29}$ Aus Paris ist er mutig gekommen, hat aber in Deutschland nichts anderes als Skeptizismus gefunden: Er war an der Musik Leverkühns auch deswegen interessiert, weil sie seiner Meinung nach eine bestimmte „qualité d'Allemand“ (DF: 583) aufweist, die im Rahmen von Konzerten o. Ä. mit neuer, internationaler Musik ein erfolgsversprechendes Alleinstellungsmerkmal darstellen könnte. Seiner Auffassung nach reiche es für Leverkühn nicht aus, dass seine Musik bei solchen Konzerten aufgeführt werde: Als Vertreter der deutschen, neuen Musik sei seine Anwesenheit ausschlaggebend. Identitätskategorien spielen in den Äußerungen von Fitelberg eine zentrale Rolle, sowohl in Bezug auf seine Selbstdarstellung als auch auf Leverkühn und dessen Musik sowie auf seine Vorstellung davon, wie er Leverkühns Musik dem Pariser Publikum präsentieren möchte.

Im zweiten Teil des Kapitels - d. h.: sobald klar wird, dass sein Angebot keine Aussichten auf Erfolg hat - kritisiert Fitelberg den Komponisten hemmungslos und äußert sich daneben über den aufkeimenden Antisemitismus, indem er selbst nationale bzw. ethnische Stereotypen anspricht:

\footnotetext{
${ }^{27}$ Siehe DF: 584.

${ }^{28}$ Vgl. DF: 589.

${ }^{29}$ Siehe Marquardt: Der Manager als Sündenbock, S. 573.
} 
Sie wissen wohl gar nicht, maître, wie deutsch Ihre répugnance ist, die sich, wenn Sie mir erlauben, en psychologue zu sprechen, aus Hochmut und Inferioritätsgefühlen charakteristisch zusammensetzt, aus Verachtung und Furcht [...]. Zwar ist es ein deutscher Aberglaube, daß es draußen nur Valse brillante gibt und Ernst nur in Deutschland. [...] Wir Juden haben alles zu fürchten vom deutschen Charakter, qui est essentiellement anti-sémitique. (DF: 589f.)

Und später:

Die Deutschen sollten es den Juden überlassen, pro-deutsch zu sein. Sie werden sich mit ihrem Nationalismus, ihrem Hochmut, ihrer Unvergleichlichkeitspuschel, ihrem $\mathrm{Ha}$ auf Einreihung und Gleichstellung [...] - sie werden sich damit ins Unglück bringen [...]. Die Deutschen sollten dem Juden erlauben, den médiateur zu machen zwischen ihnen und der Gesellschaft [...]. (DF: 591f.)

Fitelberg scheint hier Leverkühn aus mindestens zwei Gründen „den médiateur“ machen zu wollen: Einerseits als Impresario, um sich für die Verbreitung von Leverkühns Werk in der Gesellschaft einzusetzen (und somit auch selbst zu profitieren), andererseits aus politischen Gründen, um zu zeigen, dass eine Kooperation zwischen einem Deutschen und einem Juden doch noch möglich ist. Es mag nicht wundern, dass er hinsichtlich der politischen Situation Deutschlands von „Valse brillante" spricht und so zugleich auf seine Kenntnisse im musikalischen Bereich hinweist: Aus dem ganzen Fitelberg-Kapitel kristallisiert sich die starke Verflechtung ethnisch-nationaler und beruflicher Identitätskategorien heraus. Auch der ständige Sprachwechsel verdeutlicht das: In Fitelbergs Worten profiliert sich der Versuch einer produktiven Mediation zwischen der deutschen und der französischen Sprache, es geht auch um die Beziehung zwischen der deutschen und der französischen Bevölkerung, die in dieser Zeit (1923) ebenfalls angespannt war. ${ }^{30}$

Um zu Marquardts Auffassung zurückzukehren, könnte aus Fitelberg in der Narration ein Sündenbock sowohl aufgrund seiner jüdischen Herkunft als auch aufgrund seines Berufs, der - wie das Zitat aus Marcello zeigt - ebenfalls Stereotypen ausgesetzt war, werden. Er handelt, obwohl er natürlich mit Leverkühns Musik Geld verdienen möchte, immer noch im Interesse der Musik. „Es gibt zwei Seelen in Fitelbergs Brust: den Geschäftsmann und den Kunstkenner“, 31 meint Darmaun. Er bezieht sich damit auf das Wesen eines Impresario bzw.

\footnotetext{
${ }^{30} \mathrm{Zu}$ einer intersektional angelegten Analyse von Sprachdifferenz siehe auch: Radaelli, Giulia: „Worte des Fremden“. Stimme und Sprachdifferenz in Elias Canettis Die Stimmen von Marrakesch. Aufzeichnungen nach einer Reise. In: Klein u. Schnicke (Hrsg.): Intersektionalität und Narratologie, S. 185-204.

${ }^{31}$ Darmaun: Thomas Mann, Deutschland und die Juden, S. 245.
} 
einer Impresaria: Ein Musikagent bzw. eine Musikagentin hat mit Konten sicherlich viel zu tun, ist aber zugleich sehr bewandert im musikalischen Bereich, ${ }^{32}$ sodass er oder sie manchmal für die „Durchsetzung echter Kunst“33 bankrott stirbt. Fitelberg leugnet nicht, dass sein erstes Projekt, das „Théâtre des fourberies gracieuses“ (DF: 580), gescheitert ist. Man könnte zudem an seinen Kompetenzen zweifeln und sich fragen, ob ein Jahr an der Sorbonne ${ }^{34}$ und die Kenntnisse berühmter Persönlichkeiten des damaligen Pariser Kulturlebens ausreichend seien, um als Kunstkenner bezeichnet zu werden.

Ein Teil der Doktor Faustus-Forschung betrachtet Fitelberg als Verführer. So vertritt beispielsweise Schmidt-Schütz anhand des Mantel-Zitats die Auffassung, ${ }^{35}$ dass die Diktion ,bereits klar [mache], daß der Konzertagent sich in die Gruppe der ,Verführer'-Gestalten“36 einreihe. Und Evelyn Cobley fügt hinzu: Der Komponist ,cannot be tempted by the world when it visits him in the guise of Saul Fitelberg". Wie bereits antizipiert, gehört allerdings auch Frau von Tolna zum „Kapitel ,Welt““ (DF: 575) von Leverkühns Biographie und von der lässt sich der fiktive Komponist doch finanzieren. Und zwar schon vor Fitelbergs Erscheinen in Pfeiffering: Das Angebot des Impresarios anzunehmen, wäre ein Verstoß gegen die Bedingungen des Finanzierungsverhältnisses. In dieser Hinsicht kann Fitelberg also als Verführer betrachtet werden. Dem Vergleich zwischen Fitelberg und Frau von Tolna widmet sich der nächste Unterabschnitt.

\subsubsection{Die ungarische Mäzenin und die Frage nach der Finanzierung des Musikwerkes}

Es gilt nun, die beiden Figuren, Saul Fitelberg und Frau von Tolna, näher zu analysieren und auf ihre Funktion im Roman einzugehen. Innerhalb der Ordnung des

\footnotetext{
${ }^{32}$ Toelle erklärt, die berühmtesten Impresari in Mailand, Venedig oder Parma seien oft ausgebildete Tänzer*innen oder Sänger*innen gewesen. Siehe Toelle: Oper als Geschäft, S. $20 \mathrm{ff}$.

${ }^{33}$ Ebd.

${ }^{34}$ Siehe DF: 580.

35 ,Und dennoch, figurez-vous, bin ich gekommen, Sie zu entführen, Sie zu vorübergehender Untreue zu verführen, Sie auf meinem Mantel durch die Lüfte zu führen und Ihnen die Reiche dieser Welt und ihre Herrlichkeit zu zeigen, mehr noch, sie Ihnen zu Füßen zu legen..." (DF: 579). Man sieht hier die Anspielung auf die Versuchung Christi durch den Teufel.

${ }^{36}$ Schmidt-Schütz: Doktor Faustus zwischen Tradition und Moderne, S. 145. Siehe auch Bergsten: Untersuchungen, S. 32.
} 
Romans scheint es kein Zufall zu sein, dass der Musikagent gleich nach dem 36. Kapitel vorgestellt wird, das von der reichen Mäzenin handelt. ${ }^{37}$ Die Identität der Frau von Tolna stellt bis heute ein Rätsel für die Doktor Faustus-Forschung dar obwohl der Großteil der Forscher*innen sie für Hetaera Esmeralda hält, wofür es auch diverse Argumente gibt: ${ }^{38}$ Zunächst die im Esmeralda-Kapitel dieser Arbeit genannten Eigenschaften einiger Schmetterlinge, nämlich die Unsichtbarkeit und die Flüchtigkeit. ${ }^{39}$ Frau von Tolna ist eine große Verehrerin Leverkühns, die sein Werk und die Aufführungen seiner Kompositionen finanziell unterstützt und seine Wirkungsstätte besichtigt, ohne jemals von ihm gesehen zu werden. ${ }^{40}$ Die Unsichtbarkeit $^{41}$ ist sowohl die Haupteigenschaft dieser Figur als auch Bedingung für das Unterstützungsverhältnis zu Leverkühn: So entsteht eine Verbindung mit den Schmetterlingen in Jonathan Leverkühns Büchern, die so gut getarnt waren, dass man sie für Blätter halten könnte. Ein anderes Argument wäre die angegriffene Gesundheit Frau von Tolnas, die auf die Syphilis Esmeraldas anspielen könnte:

Sie lebte in Paris, Neapel, Ägypten, im Engadin, von Ort zu Ort begleitet von einer Jungfer, einem männlichen Angestellten, der etwas wie einen Quartiermacher und Reisemarschall abgab, und einem allein ihren Diensten gewidmeten Arzt, was auf delikate Gesundheit schließen ließ. (DF: 568$)^{42}$

Aus dem obigen Zitat geht auch der flüchtige und mondäne Charakter von Frau von Tolnas Lebensstil hervor. So Zeitblom:

\footnotetext{
${ }^{37}$ Vgl. auch Marquardt: Der Manager als Sündenbock, S. 567.

${ }^{38}$ Das Hauptvorbild für diese Figur sei, so der Autor, Frau von Meck, die unsichtbare Freundin und Mäzenin Tschaikowskis. Puschmann schlägt ein weiteres Vorbild vor: Irene Hirsch-Hatvany, eine ungarische Aristokratin.Die Hatvanys seien Gründer der ungarischen Zuckerindustrie gewesen und sollen auch Thomas Mann kennengelernt haben; Zeitblom berichtet von den ,Zuckerrüben-Pflanzungen“ (DF: 568) in Frau von Tolnas Villa am Balaton. Vgl. Ent: 30; TB2: 31.01.1944, S. 16; Bergsten: Untersuchungen, S. 82 f.; Puschmann: Magisches Quadrat, S. 142.

${ }^{39}$ Vgl. Kap. 8 u. Börnchen: Kryptenhall, S. 205.

${ }^{40}$ Vgl. DF: $566-570$.

${ }^{41}$ Vgl. DF: 566: „Frau von Tolna aber ist eine unsichtbare Figur. Ich [Zeitblom] kann sie dem Leser nicht vor Augen stellen, von ihrem Äußeren nicht das kleinste Zeugnis geben, denn ich habe sie nicht gesehen und nie eine Beschreibung von ihr empfangen [...]“.

${ }^{42}$ Dies allerdings ist die Schlussfolgerung Zeitbloms, denn Frau von Tolna könnte auch hypochondrisch sein oder fremden Ärzten nicht vertrauen.
} 
Keinen dieser Aufenthalte, Stadthaus, Gutsschloß und Sommervilla, benutzte die Eigentümerin für irgend längere Zeit. Ganz vorwiegend, man kann sagen: fast immer, war sie auf Reisen, indem sie die Heimstätten, an denen sie offenbar nicht hing, von denen Unruhe oder peinliche Erinnerungen sie vertrieben, der Obsorge von Verwaltern und Hausmeistern überließ. (DF: 568)

Weiters werden der Ring aus „hellgrüne[m] Ural-Smaragd“ (DF: 569), den Leverkühn zusammen mit dem ersten Brief bekommt ${ }^{43}$ sowie die ungarische Herkunft der Mäzenin erwähnt. Zeitblom präzisiert die Übersetzung des Namens ,Pressburg', „Poszony“ (DF: 225), ins Ungarische, wo die intime Begegnung mit der Prostituierten stattfindet. Auf diese Weise schafft er einen symbolischen Zusammenhang, der sich auf die Bedeutung des Namens ,Esmeralda' sowie auf das Land Ungarn stützt. ${ }^{44}$ Schließlich hätte Esmeralda (und das könnte eine Erklärung für den Nachnamen bieten) einen reichen Aristokraten heiraten können, Herrn von Tolna, der jedoch beim Pferderennen tödlich verunglückt war. ${ }^{45}$

Es gibt auch Argumente, die gegen diese Identifikation sprechen. Was die Anspielung auf den Schmetterling angeht, könnte man darauf hinweisen, dass diese Insekten „ein ephemeres Leben fristen“ (DF: 26). Die intime Begegnung mit Esmeralda lässt sich auf Mai 1906 datieren, ${ }^{46}$ wo sie bereits krank ist. 1927 - zur Zeit der Uraufführung der Apocalipsis cum figuris sowie der ersten Schilderung der Figur Frau von Tolnas ${ }^{47}$ müsste sie also aus einer zeitlogischen, die Intradiegese berücksichtigenden Perspektive schon längst tot sein. Darüber hinaus trägt der geschenkte Ring - laut Zeitblom ,,Symbol der Bindung, der Fessel, ja der Hörigkeit“ (DF: 570) - „die Anfangsworte eines Apollon-Hymnus des Kallimachos“ (DF: 570). Der Verweis auf den Gott Apoll, Schützer der Künste, bestätigt die Auffassung eines Verhältnisses ,im rein Geistigen“ (DF: 571) zwischen dem Komponisten und der Verehrerin, das wenig Dionysisches enthält. Unter diesem Aspekt ist die Verbindung Frau von Tolnas mit Esmeralda fragwürdig, denn im Fall der Prostituierten handelt es sich um eine andere Art von Beziehung. Ein letztes zu betrachtendes Element hat mit der Darstellung Esmeraldas als Femme fatale durch Zeitblom zu tun. Oft wird diese symbolische Figur, nicht selten im Namen der Kunst, geopfert. ${ }^{48}$ Wenn also die verschleierte Frau, die bei der Beerdigung

\footnotetext{
${ }^{43}$ Vgl. Oswald: The Enigma, S. 252.

${ }^{44}$ Siehe auch ebd., S. 251.

${ }^{45}$ Vgl. DF: 567.

${ }^{46}$ Vgl. DF: 224.

${ }^{47}$ Die Briefe Frau von Tolnas hatte Leverkühn schon vor der Uraufführung erhalten, aber sie ist zu diesem Zeitpunkt sicherlich noch lebendig.

${ }^{48}$ Vgl. Hilmes: Kleopatra, S. 115 f.; Wurz: Kundry, Salome, Lulu, S. 38.
} 
Leverkühns erscheint, zugleich Esmeralda und Frau von Tolna ist, wäre daraus zu schließen, dass in Doktor Faustus die Femme fatale nicht geopfert wurde. Dies wäre im Sinne einer Erneuerung des Typus durchaus möglich; zweifelsohne wird allerdings Frau von Tolna nicht als Femme fatale, sondern vielleicht eher als Femme fragile dargestellt.

Abgesehen von der Identitätsproblematik verkörpern Saul Fitelberg und die ungarische Witwe zwei antithetische Formen und Konzeptionen des Musikbetriebs, die in Adornos Philosophie geschildert werden. Der Philosoph schreibt dazu:

Die nicht konformierende Musik ist vor solcher Vergleichgültigung des Geistes, der des Mittels ohne Zweck, nicht geschützt. Wohl bewahrt sie ihre gesellschaftliche Wahrheit kraft der Antithese zur Gesellschaft, durch Isolierung, aber diese läßt wiederum auch sie selber verdorren. Es ist, als wäre ihr der Stimulus zur Produktion, ja die raison d'être entzogen. [...] Unter den Symptomen solcher Lähmung ist das sonderbarste vielleicht, daß die fortgeschrittene Musik, welche durch Autonomie eben jenes demokratisch breite Publikum von sich stieß, das sie einmal durch Autonomie erobert hatte, nun an die Ära vor der bürgerlichen Revolution zugehörige und in ihrem Wesen Autonomie gerade ausschließende Einrichtung der Auftragskomposition sich erinnert. [...] Fast alle exponierten Stücke, die überhaupt noch fertig werden, sind nicht auf dem Markt verkäuflich, sondern von Mäzenen oder Institutionen bezahlt. Der Konflikt zwischen Auftrag und Autonomie kommt in widerwilliger, stockender Produktion an den Tag. Denn weit mehr noch als in der absolutistischen Ära sind heute der Mäzen und der Künstler, die immer schon in prekärem Verhältnis standen, einander fremd. Der Mäzen hat keinerlei Beziehung zum Werk, sondern bestellt es als einen Sonderfall jener „kulturellen Verpflichtung", die selber bloß die Neutralisierung der Kultur bekundet; für den Künstler aber reicht die Festlegung auf Termine und bestimmte Gelegenheiten schon hin, um die Unwillkürlichkeit, deren das emanzipierte Ausdrucksvermögen bedürfte, zu tilgen. Es herrscht historisch prästabilierte Harmonie zwischen der materiellen Nötigung zur Auftragskomposition durch Unverkäuflichkeit und einem Nachlassen der inneren Spannung, das zwar den Komponisten dazu befähigt, mit der in unbeschreiblicher Anstrengung errungenen Technik des autonomen Werkes heteronome Aufgaben zu erfüllen, dafür aber vom autonomen Werk ablenkt“. (PhnM: 28f.)

Der „Konflikt zwischen Auftrag und Autonomie“ kommt auch in Doktor Faustus durch die zwei Figuren zum Tragen. Fitelberg bietet Leverkühn Reichtum und Popularität an. Laut Adorno ist aber die radikale Musik ,die Antithese gegen die Ausbreitung der Kulturindustrie über ihr [sic] Bereich“ (PhnM: 15), sie macht „spröde gegen die ratio der Verkäuflichkeit“ (ebd.): Die Welt der Impresari lässt sich folglich schwer mit ihr vereinbaren, denn diese Form der Musikfinanzierung ist vom Geschmack des Publikums nie komplett unabhängig. Auch wenn Impresari wie Fitelberg für keine bekannten Operntheater arbeiten, sondern sich 
für die Neue Musik einsetzen, müssen sie sich bestimmten Bedingungen des Marktes beugen: Die bloße Aufführung des Werkes in Abwesenheit seines*seiner Urheber*in ist beispielsweise nicht erfolgsversprechend, die Isolierung des*der Künstler*in kann diese Form der Musikfinanzierung nicht gewährleisten. Die Impresari wissen als Geschäftsmänner und- frauen und ebenso als Kunstkennende, wie weit sie gehen dürfen - so Toelle: ${ }^{49}$

Er[*Sie] musste das Schicksal berechnen können und möglichst alle Zufälle ausschalten, die Erfolg oder Misserfolg einer Produktion oder eines Abends beeinflussen konnten, und er[*sie] musste die Reputation, die Qualität, den Wert und die Erfolgsaussichten des zu erwartenden spettacolo mit der Situation am Theater und in der Stadt und den Erwartungen des Publikums in Beziehung setzen.

Adorno vertritt außerdem die These, Mäzen*innen und Künstler*innen seien „weit mehr noch als in der absolutistischen Ära [...] einander fremd“ (PhnM: 29). Dieses Konzept wird im Roman prosopoietisch durch die unsichtbare Verehrerin Leverkühns realisiert, die diese neue Form des distanzierten Mäzenatentums verkörpert. Dennoch zeichnet sie nicht in toto Adornos Idee nach: Frau von Tolna scheint aus keiner „kulturellen Verpflichtung“ heraus Leverkühns Schaffen zu finanzieren, da sie sein Werk sehr genau kennt und alle Aufführungen besucht. Die Auffassung aber einer scheinbar größeren Autonomie kraft der Wahl des Mäzenatentums könnte durch den Ring kaum deutlicher werden: Künstler und Mäzenin, nicht zufällig jeweils männlichen und weiblichen Geschlechts, schließen einen künstlerischen Vertrag ab, der an einen Ehevertrag mit seinen Implikationen ewiger Treue denken lässt. Komplett frei ist Leverkühn somit nicht, auch wenn im Roman nie erwähnt wird, dass Frau von Tolna auf irgendeine Art und Weise Stil und Sujets seiner Produktion zu beeinflussen versucht: Als Zeichen seiner Dankbarkeit gegenüber und zugleich aufgrund der Bindung an die Mäzenin trägt Leverkühn während der Komposition der Apocalipsis den Ring die ganze Zeit über. ${ }^{50}$

Obwohl Zeitblom versucht, Frau von Tolna als „dienende Frau“ Leverkühns und zugleich auch unter gewissen Aspekten als Femme fragile (z. B.: die delikate Gesundheit, die Unsichtbarkeit) darzustellen, wird deutlich, dass es in Doktor Faustus wahrscheinlich keine mächtigere weibliche Figur als sie gibt. Sie hat sowohl das Geld als auch verfügt sie über die richtigen Kontakte zu Verlagen und Konzertveranstalter*innen, um Leverkühns Werk ökonomisch fördern zu können. Es liegt diesbezüglich eine Subversion von Geschlechterverhältnissen vor, die

\footnotetext{
${ }^{49}$ Toelle: Oper als Geschäft, S. 30.

${ }^{50}$ Siehe DF: 570.
} 
dadurch noch deutlicher wird, dass die Mäzenin, also die Frau, dem Komponisten, also dem Mann, den Ring schenkt. Leverkühn ist sich über die Bedingungen eines solchen exklusiven Verhältnisses im Klaren und lässt sich von Fitelberg nicht verführen.

Schließlich gilt es an dieser Stelle auf eine Dichotomie einzugehen, die sich im Roman profiliert, nämlich die Dichotomie Frankreich - Ungarn. Es mag bereits aufgefallen sein, dass Frankreich, und insbesondere Paris, einen unrealisierbaren Traum darstellt. Marie Godeau lebt in der französischen Hauptstadt, will aber Leverkühn nicht heiraten. Schwerdtfeger wird, kurz bevor er nach Paris umziehen soll, getötet. Und der Impresario aus Paris beabsichtigt, Leverkühns Musik in diversen Städten zu präsentieren - u. a. auch in Paris - und den Komponisten in die dortigen Zirkel von Intellektuellen einzuführen. Sein Angebot wird abgelehnt. ${ }^{51}$ Ganz im Gegenteil dazu stellt Ungarn einen erfüllbaren Traum dar. Leverkühn begegnet der Prostituierten in Leipzig, findet sie dann in der (damals: Monarchie Österreich-Ungarn) ungarischen Stadt Pressburg wieder. Frau von Tolna kommt aus demselben Land wie Esmeralda: In ihrem Schloss verbringt Leverkühn zwölf Tage (wieder die Zahl zwölf, die an die Zwölftontechnik und an die Zahl zwei erinnert ${ }^{52}$ mit Schwerdtfeger, was Zeitblom als „leicht exzentrische[] Episode“ (DF: 574) bezeichnet. Nationalbedingte Formen des kulturellen Lebens, das liberale Paris vs. das traditionsgebundene Ungarn, treten durch die zwei Figuren Frau von Tolna und Saul Fitelberg zutage; zudem wird, worauf Schmidt-Schütz hinweist, im Fitelberg-Kapitel die Stadt Paris auch mit Pfeiffering kontrastiert. ${ }^{53}$ Das bayerische Dorf, das der Impresario wohl mit Absicht unzutreffend als „Idyll“ (DF: 578) bezeichnet, hat mit der französischen Metropole in den 1920er Jahren, wahrscheinlich das damalige kulturelle Zentrum Europas, wo man Veranstaltungen aller Art besuchen und auf Picasso, Satie, Joyce, Miller, usw. treffen konnte, wenig gemein, auch unter politischen Gesichtspunkten nicht. Zusammenfassend liegt es nahe, dass Fitelberg hauptsächlich vor der Unverkäuflichkeit des Musikwerkes der Neuen Musik und vor dem in Pfeiffering geäußerten Misstrauen flieht. Selbst die Begründung für diese Flucht ist in einem intersektionalen Denkparadigma angesiedelt, denn sie vereint berufliche und politisch-ethnische Aspekte und weist folglich noch einmal auf die Notwendigkeit hin, diese Figur aus dieser Forschungsperspektive zu untersuchen.

\footnotetext{
${ }^{51}$ Siehe auch Darmaun: Thomas Mann, Deutschland und die Juden, S. 249.

${ }^{52} \mathrm{Vgl}$. 6.1.2.

${ }^{53}$ Vgl. Schmidt-Schütz: Doktor Faustus zwischen Tradition und Moderne, S. 174.
} 


\subsection{Vom Roman zur Musik}

Über Formen der Musikfinanzierung reflektieren nicht nur die Fitelberg-Kapitel von Doktor Faustus, sondern auch Manzonis Oper durch die Impresario-Figur Fitelberg gerade in einem der bekanntesten Operntheater der Welt: Dieser zweite Teil des Kapitels untersucht die Konsequenzen des Transfers dieser inhaltlichen Mikroform in ein anderes Medium und in einen anderen Kontext.

\subsubsection{Saul Fitelberg in Manzonis Oper: Eine Reflexion über Finanzierungsmöglichkeiten und die Zukunft der Oper}

Der Figur des jüdischen Impresarios ist in Manzonis Doktor Faustus das dritte Bild des zweiten Aktes gewidmet. In diesem Abschnitt sei zuerst der Frage nachgegangen, warum die Oper nicht alle Romanfiguren übernimmt, Saul Fitelberg aber beibehält. Sogar Serenus Zeitblom, die Erzählinstanz, bleibt am Rande von Manzonis Werk; ganz zu schweigen von Schwerdtfeger, der nicht einmal erwähnt wird. Im Gegensatz dazu lässt Manzonis Doktor Faustus Fitelberg eine ganze ca. siebenminütige Episode hindurch Adrian Leverkühn ohne Erfolg sein Angebot vorstellen. Durch ihn wagt die Oper in einem der renommiertesten Operntheater der Welt über die Möglichkeit zu reflektieren, wie man sie ökonomisch fördern könnte, und zwar zusammen mit denjenigen, die noch in die Oper gehen und sie somit auch finanzieren. Das Bild lässt sich darüber hinaus auch als Reflexion über die Musikgattung und ihre Zukunft begreifen, denn die Oper ist im Rahmen der Neuen Musik, die zum Teil der Idee der absoluten, rein instrumentalen Musik verpflichtet ist, zweifelsohne eine kontroverse Gattung. ${ }^{54}$ Sorg erklärt: „Mit Fitelberg zieht am offensichtlichsten Manzonis Kritik an einem marktorientierten Kunstbegriff in die Oper ein". ${ }^{55}$ Der Impresario sei seiner Auffassung nach als kapitalistischer Versucher porträtiert und in diesem Bild kritisiere die Oper die exzessive Vermarktung der Musik. ${ }^{56}$ Die wiederholte Verwendung des Falsetts konnotiert den Musikagenten als Lügner noch deutlicher: Das italienische Wort falsetto ist der Diminutiv vom Adjektiv falso (,falsch/unehrlich'); darüber hinaus erzeugt diese Art von Gesang einen Eindruck von Künstlichkeit. Nicht

\footnotetext{
${ }^{54}$ Die Frage nach der Zukunft der Oper ist immer noch aktuell: Vgl. Beyer, Barbara, Kogler, Susanne u. Roman Lemberg (Hrsg.): Die Zukunft der Oper. Zwischen Hermeneutik und Performativität. Berlin: Theater der Zeit 2014.

${ }^{55}$ Sorg: Beziehungszauber, S. 206.

${ }^{56}$ Vgl. ebd., S. 205.
} 
selten findet diese Gesangstechnik dann Verwendung, wenn Fitelberg die Namen berühmter Persönlichkeiten zitiert. Es wirkt so, als wolle die Oper unterstreichen, dass hier der Impresario den Höhepunkt seiner Lügen und Überredungsstrategien erreicht (Abbildung 10.1) :

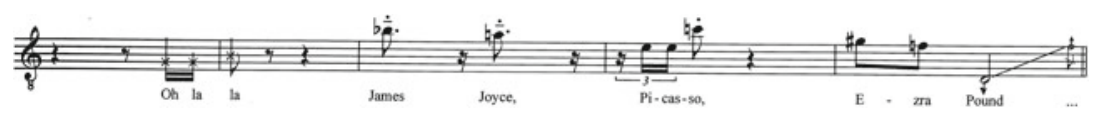

Abbildung 10.1 Die Verwendung des Falsetts - grafisch durch Punkte auf den entsprechenden Tönen signalisiert - bei der Erwähnung von Eigennamen (M-DF: 142 f., T. 275-279)

Dennoch ist die Figur des jüdischen Impresarios, der allerdings bei Manzoni lediglich als Impresario inszeniert wird (andere Identitätskategorien spielen in der Oper selten eine Rolle), auch eine komische: „Mit der Verführungsabsicht geht auch eine Komik einher" ${ }^{57}$ präzisiert Sorg. Manzonis Fitelberg scheint aus einer opera buffa zu stammen, da er sich in der Aufnahme der Uraufführung auf der Bühne ständig bewegt und sogar Leute aus Paris imitiert, wie das folgende Textbeispiel zeigt:

SF: (parlato, con un timbro di voce diverso; come mimando un altro ipotetico personaggio; con tono molto salottiero) Madame, oh madame! Que pensez vous, madame? On me dit, madame, que vous êtes fanatique de musique; tout le monde sait, madame, que votre jugement musical est infaillible!“. (M-DF: 144, T. 290) ${ }^{58}$

Genauer gesagt hat diese Szene eine Intermezzo-Qualität, weil sie sich zwischen den Echo-Bildern befindet: „Die Komik dieses dritten Bildes hellt für einen Moment die tragisch-düstere Geschichte um Leverkühns Neffen auf und besitzt damit auch eine retardierende Funktion" ${ }^{59}$ unterstreicht Sorg. ${ }^{60}$ Daher lässt sie eher an ein komisches Zwischenspiel vor dem tragischen Ende eines italienischen dramma per musica aus dem 18. Jahrhundert denken.

\footnotetext{
${ }^{57}$ Ebd., S. 208.

${ }^{58}$, ,gesprochen, mit einem anderen Timbre der Stimme; als ob er eine andere hypothetische Figur imitieren würde; mit starkem Salonton)“. Für den restlichen Text vgl. DF: 581 und $584 \mathrm{f}$.

${ }^{59}$ Sorg: Beziehungszauber, S. 208.

${ }^{60}$ Vgl. 11.2.3.
} 
Was die Sprache von Manzonis Fitelberg angeht, kann man einige kleine Unterschiede zu Manns Roman bemerken. Zum Beispiel die Dominanz des Französischen. In der Oper wird zu einem Großteil auf die Mischung zweier Sprachen, was hingegen den Roman prägt, verzichtet: Der Impresario hätte teils auf Italienisch, teils auf Französisch sprechen können. Vielleicht thematisiert die Oper aufgrund der programmatisch deklarierten ,,sgermanizzazione“61 des Romans die Problematik des Antisemitismus, der im Roman durch Fitelberg wahrscheinlich am deutlichsten hervortritt, kaum und lässt Fitelberg weder Deutsch noch Italienisch sprechen. An vereinzelten Stellen wird darauf hingewiesen, etwa „ça c'est bien allemand!“ (M-DF: 147, T. 306), „Une confusion tragique - Wagner...“ (MDF: 155, T. 361 ff.) und „Deutschland...Deutschland!“ (M-DF: 158, T. 385 ff.). Durch das Französische gewinnt aber der Bezug auf das kulturelle Leben in Frankreich an Bedeutung und Fitelberg wird eher als jüdischer Impresario denn als französischer dargestellt. ${ }^{62}$

Formal betrachtet ist außerdem Fitelbergs Episode in der Oper ein echter Monolog vom Anfang bis Ende, oder, genauer gesagt, ein Solo des Sängers: Niemand stellt den Agenten vor, er betritt plötzlich die Bühne und präsentiert sich. Und niemand kommentiert, was er sagt. Ein Grund dafür ist, dass sich Manzoni für die Verwendung der Romanpassagen in der direkten Rede entscheidet und im entsprechenden Kapitel ist er die einzige Figur, die sich auf diese Weise ausdrückt. ${ }^{63}$

Sorg unterstreicht, dass Manzoni hier mit Klangmöglichkeiten experimentiert, „,indem Geräusch und Klang zusammengeführt werden“. ${ }^{64}$ Er behauptet außerdem, die Musiksprache stehe „gegen die Verführungsversuche Saul Fitelbergs“. 65 Es scheint aber, dass die ,abgerissene[n] Motive der Streicher"66 sowie der ganze Musikverlauf hervorragend zur tragikomischen Figur passen und ihre Besonderheit noch deutlicher unterstreichen. Ein Beweis dafür ist, dass seine Rede nie vom Orchester zugedeckt, sondern einfach begleitet wird. Die Zusammenführung von Klang und Geräusch könnte sich zudem im Medium der Musik auf Fitelbergs

\footnotetext{
${ }^{61}$ Manzoni: Il linguaggio del Doktor Faustus, S. 305. Vgl. auch 5.2.1.1.

${ }^{62}$ Somit verweist die Oper aufgrund des Aufführungskontextes indirekt auf die Unterschiede zwischen italienischer und französischer Oper.

${ }^{63}$ Vgl. (sowohl für die Auswahl der Textstellen aus dem Roman als auch für die „sgermanizzazione“) 5.2.1.1.

${ }^{64}$ Sorg: Beziehungszauber, S. 205.

${ }^{65}$ Ebd.

${ }^{66}$ Ebd., S. 208.
} 
Versuch beziehen, die Neue Musik an die Welt der Impresari anzupassen, sie verkäuflicher und mondäner zu machen. Fitelbergs Angebot wird aber in der Oper komplett ignoriert: Eher als die Einsamkeit des Kunstwerkes wird hier die Einsamkeit der Welt der Impresari, die - wie Adorno in der Philosophie der Neuen Musik darlegt - mit der neuen musikalischen Situation nicht kommunizieren kann und folglich für lächerlich erklärt wird. In Manzonis Oper treten Adornos Auffassungen in umgekehrter Form auf: Nicht mit der Neuen Musik will keine*r ,etwas zu tun haben [...], die Individuellen so wenig wie die Kollektiven“ (PhnM: 126). Vielmehr ,verhallt“ (ebd.) das Angebot des Impresarios Saul Fitelberg in der Oper und im Roman ,ungehört, ohne Echo“ (ebd.) und wird dann schnell vergessen.

\subsection{Fazit}

In diesem Kapitel wurde der narratologisch-intermediale Akzent der Untersuchung um das Forschungsparadigma der Intersektionalität erweitert. Die drei Bereiche Intersektionalität, Intermedialität und Narratologie werden kombiniert, um die sozialen Ungleichheiten darzustellen, die im Roman anhand der Figur des jüdischen Impresarios Fitelberg konstruiert werden. Während in Manzonis Oper der Bezug auf Fitelbergs Herkunft, ähnlich wie im Fall von Esmeralda, in den Hintergrund rückt, so wird jedoch hier die sozial-politische Kritik der FitelbergKapitel in das neue Medium transferiert. Der Impresario dient dazu, über die Finanzierung des Musikwerkes zu reflektieren. Um sich der Analyse des intermedialen Effekts der Verstärkung des vorigen Kapitels anzuschließen, kann zu Manzonis Doktor Faustus gesagt werden, dass in dieser Oper die Verstärkung der sozial-politischen Kritik vor allem kontextuell bedingt ist: Sie thematisiert diesen Aspekt in einem der renommiertesten Operntheater der Welt und zielt folglich noch ausdrücklicher als der Roman auf eine Reflexion durch das Publikum ab. Wie Henze verstärkt auch Manzoni eine Figur aus Doktor Faustus, die sonst in der Forschungsliteratur kaum behandelt wird, was dementsprechend noch einmal ein Beweis dafür ist, dass die Auseinandersetzung mit der kompositorischen Rezeptionsgeschichte von Thomas Manns Roman eine neue Lektüre des Werkes bewirkt. 
Open Access Dieses Kapitel wird unter der Creative Commons Namensnennung - Nicht kommerziell - Keine Bearbeitung 4.0 International Lizenz (http://creativecommons.org/lic enses/by-nc-nd/4.0/deed.de) veröffentlicht, welche die nicht-kommerzielle Nutzung, Vervielfältigung, Verbreitung und Wiedergabe in jeglichem Medium und Format erlaubt, sofern Sie den/die ursprünglichen Autor(en) und die Quelle ordnungsgemäß nennen, einen Link zur Creative Commons Lizenz beifügen und angeben, ob Änderungen vorgenommen wurden. Die Lizenz gibt Ihnen nicht das Recht, bearbeitete oder sonst wie umgestaltete Fassungen dieses Werkes zu verbreiten oder öffentlich wiederzugeben.

Die in diesem Kapitel enthaltenen Bilder und sonstiges Drittmaterial unterliegen ebenfalls der genannten Creative Commons Lizenz, sofern sich aus der Abbildungslegende nichts anderes ergibt. Sofern das betreffende Material nicht unter der genannten Creative Commons Lizenz steht und die betreffende Handlung nicht nach gesetzlichen Vorschriften erlaubt ist, ist auch für die oben aufgeführten nicht-kommerziellen Weiterverwendungen des Materials die Einwilligung des jeweiligen Rechteinhabers einzuholen.

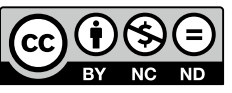

